\title{
Retinal and Optic Nerve Degeneration in Patients with Multiple Sclerosis Followed up for 5 Years
}

Elena Garcia-Martin, PhD, ${ }^{1,2}$ Jose R. Ara, PhD, ${ }^{2,3}$ Jesus Martin, PhD, ${ }^{2,3}$ Carmen Almarcegui, PhD, ${ }^{2,4}$ Isabel Dolz, PhD, ${ }^{2,4}$ Elisa Vilades, MD, ${ }^{1,2}$ Laura Gil-Arribas, PhD, ${ }^{1,2}$ Francisco J. Fernandez, PhD, ${ }^{1,2}$ Vicente Polo, PhD, ${ }^{1,2}$ Jose M. Larrosa, PhD, ${ }^{1,2}$ Luis E. Pablo, PhD, ${ }^{1,2}$ Maria Satue, PhD ${ }^{1,2}$

Purpose: To quantify retinal nerve fiber layer (RNFL) changes in patients with multiple sclerosis (MS) and healthy controls with a 5-year follow-up and to analyze correlations between disability progression and RNFL degeneration.

Design: Observational and longitudinal study.

Participants: One hundred patients with relapsing-remitting MS and 50 healthy controls.

Methods: All participants underwent a complete ophthalmic and electrophysiologic exploration and were re-evaluated annually for 5 years.

Main Outcome Measures: Visual acuity (Snellen chart), color vision (Ishihara pseudoisochromatic plates), visual field examination, optical coherence tomography (OCT), scanning laser polarimetry (SLP), and visual evoked potentials. Expanded Disability Status Scale (EDSS) scores, disease duration, treatments, prior optic neuritis episodes, and quality of life (QOL; based on the 54-item Multiple Sclerosis Quality of Life Scale score).

Results: Optical coherence tomography (OCT) revealed changes in all RNFL thicknesses in both groups. In the MS group, changes were detected in average thickness and in the mean deviation using the GDx-VCC nerve fiber analyzer (Laser Diagnostic Technologies, San Diego, CA) and in the P100 latency of visual evoked potentials; no changes were detected in visual acuity, color vision, or visual fields. Optical coherence tomography showed greater differences in the inferior and temporal RNFL thicknesses in both groups. In MS patients only, OCT revealed a moderate correlation between the increase in EDSS and temporal and superior RNFL thinning. Temporal RNFL thinning based on OCT results was correlated moderately with decreased QOL.

Conclusions: Multiple sclerosis patients exhibit a progressive axonal loss in the optic nerve fiber layer. Retinal nerve fiber layer thinning based on OCT results is a useful marker for assessing MS progression and correlates with increased disability and reduced QOL. Ophthalmology 2017;n:1-9 () 2017 by the American Academy of Ophthalmology.

The main cause of disability in patients with multiple sclerosis (MS) is axonal loss. ${ }^{1-3}$ Axonal deterioration in the retinal nerve fiber layer (RNFL) can be detected and quantified using optical coherence tomography (OCT) or scanning laser polarimetry (SLP). ${ }^{3-8}$ Typical ophthalmic findings in MS patients are optic nerve atrophy and peripapillary RNFL thinning. ${ }^{8}$

The effects of MS on the central nervous system generally are difficult to examine directly, but the RNFL, which is made up of mainly nonmyelinated retinal ganglion cell axons, provides easy access for clinical examination. The RNFL thickness can be measured to assess axons and axonal damage directly. Even in MS patients without preceding symptoms of optic neuritis show decreases in RNFL thickness. ${ }^{4-8}$ Visual evoked potential (VEP) tests also reveal abnormalities in MS patients. ${ }^{9-11}$

Optical coherence tomography has been used to measure RNFL thickness as a biologic marker of axonal damage $^{12-14}$ and to monitor disease progression in patients with MS..$^{7,8}$ In fact, some authors suggest that OCT may be an efficient and less expensive substitute for magnetic resonance imaging as a method of monitoring the disease. ${ }^{15-17}$ In this study, we compared structural and functional changes in the RNFL in MS patients with those in healthy controls. We further evaluated the correlation of the RNFL changes with disease progression and changes in quality of life (QOL) over 5 years.

\section{Methods}

\section{Participants}

This prospective longitudinal study included patients with definite relapsing-remitting MS and healthy controls with a 5-year followup. A total of 204 eyes of 102 patients and 100 eyes of 50 healthy controls were evaluated at baseline and then every year for 5 years. Multiple sclerosis was diagnosed based on the 2010 revision of the McDonald criteria and confirmed by a neurologist specializing in 


\section{Ophthalmology Volume $\mathbf{n}$, Number $\mathbf{n}$, Month 2017}

MS. ${ }^{18}$ Patients with a visual acuity less than 0.1 (Snellen scale), intraocular pressure more than $20 \mathrm{mmHg}$, active MS flare (of any neurologic deficit), or a combination thereof in the 6 months preceding their enrollment into the study or at any of the annual visits were excluded from the study. Active MS flare was considered a reason for exclusion because acute axonal loss could mask neuronal damage secondary to MS progression (i.e., chronic neurodegeneration), which was the main purpose of this study. Patients with refractive errors of more than 5 diopters of spherical equivalent refraction or 3 diopters of astigmatism also were excluded from the study.

\section{Standard Protocol Approvals, Registrations, and Patient Consents}

The study procedures were performed in accordance with the tenets of the Declaration of Helsinki, and the study protocol was approved by the local ethics committees. Written informed consent to participate in the study was obtained from all participants.

\section{Main Outcome Measures}

All participants underwent a complete neuro-ophthalmic examination, including assessment of best-corrected visual acuity using the Snellen chart, pupillary reflexes, and ocular motility; examinations of the anterior segment, intraocular pressure (IOP) with the Goldmann applanation tonometer, and papillary morphologic features by funduscopic examination; and OCT, SLP, and VEP. Each eye was considered separately. In addition, the Expanded Disability Status Scale (EDSS) score, disease duration, treatments, prior episodes of optic neuritis, and QOL (using the 54-item Multiple Sclerosis Quality of Life Scale [MSQOL-54] score) were evaluated. Every year for 5 years, patients were reexamined and changes in all parameters were recorded. An increase in the EDSS score during the follow-up was considered a sign of disease progression.

Visual acuity was measured with the Snellen chart at a distance of $6 \mathrm{~m}$ and best-corrected monocular vision. The papilla morphologic features were assessed with a 78-diopter lens and classified as normal, diffuse, or sectorial atrophy or edema.

Peripapillary RNFL and macular volume measurements were obtained using the Cirrus 3000 HD OCT (Carl Zeiss Meditec, Jena,
Germany). The same experienced operator (E.V.) performed all scans, and no manual correction was applied to the OCT output. An internal fixation target was used to ensure the highest reproducibility, and poor-quality scans were rejected before analysis of the data. For the Cirrus OCT scans, image quality was assessed based on the signal strength measurement, which is a combination of the signal-to-noise ratio and the uniformity of the signal within a scan (scale of $1-10$, where 1 is categorized as poor image quality and 10 as excellent image quality). We analyzed only those images with a score of more than 7. The Cirrus OCT optic disc protocol generates $200 \times 200$ cube images with 200 lineal scans that enable analysis of a $6-\mathrm{mm}^{3}$ area around the optic nerve, and the Cirrus OCT macular cube $512 \times 128$ protocol provides macular volume measure. For each scan series, mean RNFL thickness, quadrant RNFL thicknesses (superior, inferior, temporal, and nasal), and macular volume were assessed using Cirrus software version 5.0.

Cirrus segmentation analysis for retinal layers also provides measurements of ganglion cell layer (GCL) thickness (evaluating 6 areas of the macular cube: superior, superonasal, inferonasal, inferior, inferotemporal, and superotemporal sectors) and measurements of the mean and minimum GCL plus inner plexiform layer value of a set of 360 spokes, where each average represents the mean number of the pixels along the spoke that lies within the measurement annulus. The minimum was selected because the thinnest portion of the GCL plus inner plexiform layer in the perifoveal region is considered to indicate the status of the ganglion cells (the most important cells in the visual pathway).

The GDx-VCC (Laser Diagnostic Technologies, San Diego, CA), consisting of a confocal scanning laser ophthalmoscope, was used to determine RNFL thickness by directing polarized light onto the Henle fiber layer and assessing 5 parameters: nerve fiber indicator, temporal-superior-nasal-inferior-temporal (TSNIT) average, superior average, inferior average, and TSNIT standard deviation.

Study investigators recorded VEPs, with electrodes fixed (with collodion) at the midoccipital and midfrontal locations and $\mathrm{Cz}$ (Z-center is the equidistant point between both ear points) as ground, using a Neuronic SenseWitness 4.0 device (Neuronic, Zaragoza, Spain) in a dark room and with full-refraction correction if necessary. Monocular stimulation and visual stimuli followed a checkerboard pattern (contrast, 80\%; check size, 47 feet; mean luminance, $93 \mathrm{~cd} / \mathrm{m}^{2}$ ) and reversed contrast patterns with a

Table 1. Epidemiologic and Neurologic Parameters Obtained at Baseline and 5-Year Examinations in Multiple Sclerosis Patients and in Healthy Controls

\begin{tabular}{|c|c|c|c|c|}
\hline & \multicolumn{2}{|c|}{ Multiple Sclerosis Patients $(\mathrm{n}=100)$} & \multirow{2}{*}{$\begin{array}{c}\text { Healthy Controls }(n=50), \\
\text { Basal Exploration }\end{array}$} & \multirow[b]{2}{*}{$P$ Value } \\
\hline & Basal Exploration & 5-Year Exploration & & \\
\hline Age (yrs) & $41.12(11.45)$ & $46.11(11.40)$ & $41.40(13.33)$ & $0.145^{*}$ \\
\hline Gender (female:male) & $68: 32$ & $68: 32$ & $35: 15$ & $0.223 *$ \\
\hline Intraocular pressure $(\mathrm{mmHg})$ & 14.26 & 14.98 & 14.77 & $0.452 *$ \\
\hline MS disease duration (yrs) & $9.26(6.68)$ & $14.25(6.67)$ & - & $<0.001^{\dagger}$ \\
\hline EDSS score & $2.59(2.13)$ & $2.65(2.14)$ & - & $0.016^{\dagger}$ \\
\hline EDSS visual function score & $1.00(1.20)$ & $0.98(1.15)$ & - & $0.098^{\dagger}$ \\
\hline MSQOL-54 score & $60.04(21.55)$ & $56.76(24.65)$ & - & $0.003^{\dagger}$ \\
\hline Eyes with history of ON, n (\%) & $51(25.5)$ & $51(25.5)$ & - & $0.901^{\dagger}$ \\
\hline Time since last $\mathrm{ON}$ episode (yrs) & $7.74(6.28)$ & $12.69(6.98)$ & - & $<0.001^{\dagger}$ \\
\hline
\end{tabular}

EDSS = Expanded Disability Status Scale; MS = multiple sclerosis; MSQOL-54 = 54-item Multiple Sclerosis Quality of Life Scale; ON = optic neuritis Boldface values indicate a statistically significant difference.

Data are mean (standard deviation) unless otherwise indicated.

*Multiple sclerosis patients versus controls.

${ }^{\dagger}$ Multiple sclerosis patients versus multiple sclerosis patients at 5 years. 


\section{Garcia-Martin et al • Progressive Degeneration in MS}

frequency of $1 \mathrm{~Hz}$. Latency and peak-to-peak amplitude were recorded for the P100 wave. We obtained at least 2 records of 100 events and calculated the mean amplitude.

The MSQOL-54 is a multidimensional health-related measure of QOL that combines generic and MS-specific items into a single instrument. ${ }^{19}$ This test is based on the 36-item Short Form Health Survey, and 18 items were added by the test developer to assess MS-specific issues, such as fatigue and cognitive function. The resultant 54-item instrument generates 12 subscales along with 2 summary scores and 2 additional single-item measures. The subscales are physical function, role limitations-physical, role limitations-emotional, pain, emotional well being, energy, health perceptions, social function, cognitive function, health distress, overall QOL, and sexual function. All dimension scores were transformed linearly to a 0 -to-100 scale. The summary scores are the physical health composite summary and the mental health composite summary; in addition, a global index score was computed as the mean of the dimension scores. Single-item measures are sexual satisfaction and change in health. The MSQOL-54 is considered both valid and reliable and is accepted widely to assess QOL accurately. ${ }^{19}$ The validity of the test also has been confirmed in European patients, ${ }^{19}$ and it is currently the most frequently used MS-specific questionnaire. ${ }^{19-21}$

\section{Statistical Analysis}

Data were analyzed using SPSS software version 20.0 (SPSS, Inc., Chicago, IL). The Kolmogorov-Smirnov test was used to assess sample distribution. The changes registered in MS and healthy participants over the 5-year study period were parametrically distributed and thus compared using a paired Student $t$ test. Changes detected in the MS patients were compared with those detected in healthy controls using a Student $t$ test. Values of $P<0.05$ were considered to indicate statistical significance; Bonferroni's correction for multiple comparisons was applied and a $P$ value of less than 0.009 was considered statistically significant. The linear correlation between changes in the structural and functional parameters of the RNFL and changes in the MSQOL-54 or EDSS scores was determined using Pearson's correlation coefficient. Regression analysis was performed to identify basal RNFL parameters that were predictors of QOL or reduced functional ability in MS patients. Changes in the ophthalmologic parameters, QOL, and disability parameters over the 5-year follow-up were compared between patients with and without prior optic neuritis using a Student $t$ test and with healthy controls by analysis of variance. Finally, following the Advised Protocol for OCT Study Terminology and Elements recommendations for

Table 2. Functional and Structural Parameters Obtained at Baseline and 5-Year Examinations in Multiple Sclerosis Patients and in Healthy

Controls and Comparison of the Change in Each Group and between Both Groups (Multiple Sclerosis Patients vs. Healthy Controls)

\begin{tabular}{|c|c|c|c|c|c|c|c|c|c|}
\hline & \multicolumn{4}{|c|}{ Multiple Sclerosis Patients $(\mathrm{n}=200$ Eyes $)$} & \multicolumn{4}{|c|}{ Healthy Controls ( $\mathrm{n}=100$ Eyes) } & \multirow{2}{*}{$\begin{array}{c}\text { P Value (Change } \\
\text { in Multiple Sclerosis } \\
\text { Patients vs. Healthy } \\
\text { Controls) }\end{array}$} \\
\hline & Baseline & 5 Years & $\begin{array}{l}\text { Annual } \\
\text { Change }\end{array}$ & P Value & Baseline & 5 Years & $\begin{array}{l}\text { Annual } \\
\text { Change }\end{array}$ & P Value & \\
\hline BCVA & $0.91(0.22)$ & $0.90(0.19)$ & -0.002 & 0.340 & $0.98(0.04)$ & $0.99(0.02)$ & 0.002 & 0.549 & 0.094 \\
\hline Ishihara test & $18.06(3.78)$ & $17.89(3.11)$ & -0.034 & 0.439 & $19.40(2.81)$ & $19.38(2.74)$ & -0.004 & 0.699 & 0.122 \\
\hline Visual field, MD (dB) & $-3.05(2.67)$ & $-3.11(3.40)$ & 0.012 & 0.441 & $0.31(1.41)$ & $0.29(1.31)$ & -0.004 & 0.105 & 0.550 \\
\hline \multicolumn{10}{|l|}{ OCT RNFL thicknesses } \\
\hline Average $(\mu \mathrm{m})$ & $94.82(17.45)$ & $86.61(14.90)$ & -1.642 & $<0.001 *$ & $98.21(10.50)$ & $95.79(9.53)$ & -0.484 & 0.032 & $<0.001 *$ \\
\hline Superior $(\mu \mathrm{m})$ & $115.95(22.01)$ & $104.34(21.04)$ & -2.322 & $<0.001 *$ & $122.49(16.93)$ & $120.27(14.93)$ & -0.444 & 0.035 & $<0.001 *$ \\
\hline Nasal $(\mu \mathrm{m})$ & $74.34(18.99)$ & $68.11(14.33)$ & -1.246 & $<0.001 *$ & $78.50(21.76)$ & $76.59(10.70)$ & -0.382 & 0.044 & $<0.001^{*}$ \\
\hline Inferior $(\mu \mathrm{m})$ & $120.89(23.17)$ & $111.34(23.63)$ & -1.910 & $<0.001 *$ & $126.10(18.36)$ & $124.73(17.94)$ & -0.274 & 0.075 & $<0.001^{*}$ \\
\hline Temporal $(\mu \mathrm{m})$ & $64.57(19.67)$ & $53.65(13.13)$ & -2.184 & $<0.001^{*}$ & $68.14(9.82)$ & $65.87(9.56)$ & -0.454 & 0.028 & $<0.001^{*}$ \\
\hline Macular volume $\left(\mathrm{mm}^{3}\right)$ & $6.55(0.33)$ & $6.43(0.29)$ & -0.024 & $<0.001 *$ & $8.29(0.44)$ & $8.23(0.55)$ & -0.012 & $0.009^{*}$ & $<0.001 *$ \\
\hline \multicolumn{10}{|l|}{ OCT GCL analysis $(\mu \mathrm{m})$} \\
\hline Average IPL+GCL & $78.45(3.55)$ & $74.89(3.71)$ & -0.712 & $<0.001 *$ & $83.12(3.20)$ & $81.56(3.43)$ & -0.312 & 0.079 & $<0.001 *$ \\
\hline Minimum IPL+GCL & $79.09(3.07)$ & $77.55(3.70)$ & -0.308 & 0.209 & $81.23(3.69)$ & $80.00(3.11)$ & -0.246 & 0.106 & 0.065 \\
\hline Fovea & $252.97(7.49)$ & $248.97(8.87)$ & -0.800 & $<0.001^{*}$ & $256.12(7.98)$ & $253.38(8.21)$ & -0.548 & 0.014 & $<0.001 *$ \\
\hline Inferior & $80.73(3.28)$ & $78.16(2.95)$ & -0.514 & 0.011 & $82.45(3.33)$ & $80.66(2.98)$ & -0.358 & 0.056 & 0.125 \\
\hline Inferonasal & $82.97(3.98)$ & $81.08(4.06)$ & -0.378 & 0.123 & $84.54(4.34)$ & $82.32(3.45)$ & -0.444 & 0.035 & 0.354 \\
\hline Nasal & $84.73(3.86)$ & $81.01(3.99)$ & -0.744 & $<0.001^{*}$ & $85.25(4.21)$ & $83.22(3.87)$ & -0.406 & 0.078 & 0.512 \\
\hline Superior & $80.95(3.87)$ & 77.49 (3.73) & -0.692 & 0.007 & $83.21(3.66)$ & $80.98(3.27)$ & -0.446 & 0.028 & 0.034 \\
\hline Inferotemporal & $78.81(3.76)$ & $74.33(3.41)$ & -0.896 & $<0.001 *$ & $82.02(3.41)$ & $80.56(3.70)$ & -0.292 & 0.230 & $0.001 *$ \\
\hline Temporal & $78.86(3.60)$ & $74.12(4.00)$ & -0.948 & $<0.001^{*}$ & $81.11(3.93)$ & $79.82(4.11)$ & -0.258 & 0.177 & 0.003 \\
\hline \multicolumn{10}{|l|}{$\operatorname{SLP}(\mu \mathrm{m})$} \\
\hline NFI & $25.15(8.53)$ & $25.45(8.09)$ & 0.060 & 0.145 & $18.70(6.87)$ & $19.04(7.80)$ & 0.068 & 0.349 & 0.975 \\
\hline TSNIT average & $51.87(10.22)$ & $50.87(11.65)$ & -0.020 & 0.045 & $52.33(6.01)$ & $52.61(7.71)$ & 0.056 & 0.451 & 0.623 \\
\hline Superior average & $62.14(12.43)$ & $62.43(11.19)$ & 0.058 & 0.137 & $65.32(5.98)$ & $64.87(5.50)$ & -0.090 & 0.559 & 0.458 \\
\hline Inferior average & $59.65(9.48)$ & $59.34(11.50)$ & -0.062 & 0.299 & $65.00(7.09)$ & $64.76(6.96)$ & -0.048 & 0.683 & 0.872 \\
\hline TSNIT SD & $21.95(5.03)$ & $23.14(5.44)$ & 0.238 & 0.016 & $20.03(4.21)$ & $20.61(4.16)$ & 0.116 & 0.078 & 0.055 \\
\hline \multicolumn{10}{|l|}{ VEP } \\
\hline Amplitude (mV) & $11.22(2.71)$ & $10.89(3.33)$ & -0.066 & 0.103 & $14.54(2.67)$ & $14.49(2.55)$ & -0.010 & 0.449 & 0.088 \\
\hline Latency (ms) & $116.33(10.21)$ & $124.03(11.02)$ & 1.540 & $0.009^{*}$ & $102.03(7.49)$ & $101.89(6.90)$ & -0.028 & 0.618 & 0.014 \\
\hline
\end{tabular}

$\mathrm{BCVA}=$ best-corrected visual acuity; $\mathrm{GCL}=$ ganglion cell layer; $\mathrm{IPL}=$ inner plexiform layer; $\mathrm{MD}=$ mean deviation; $\mathrm{NFI}=$ nerve fiber indicator; OCT $=$ optical coherence tomography; RNFL = retinal nerve fiber layer; SD = standard deviation; SLP $=$ scanning laser polarimetry; TSNIT $=$ temporal-superior-nasal-inferior-temporal; VEP = visual evoked potential.

Data are mean $(\mathrm{SD})$ unless otherwise indicated. $P<0.05$ was considered to indicate statistical significance.

* Statistical significance using Bonferroni's correction for multiple comparisons. 


\section{Ophthalmology Volume - Number $\mathbf{n}$, Month 2017}

reporting quantitative OCT studies, ${ }^{22}$ comparison of the change registered during the 5-year follow-up between patients and controls was repeated using the mean of eyes with nonoptic neuritis in the MS group.

\section{Results}

A total of 204 eyes of 102 MS patients (mean age, $41.12 \pm 11.45$ years) and 100 eyes of 50 healthy controls (mean age, $41.40 \pm 13.33$ years) were included in the study. The female-tomale ratio was $3: 1$ in both groups (70 women and 32 men in the MS group, and 35 women and 15 men in controls group). Age, gender, and intraocular pressure were not significantly different between groups $(P=0.145,0.223$, and 0.452 , respectively). Two patients progressed from relapsing-remitting MS type to secondary progressive type during the study, and the data for these patients were excluded from the final statistical analysis.

The mean EDSS score was $2.59 \pm 2.13$ at the baseline examination and $2.65 \pm 2.14$ at the 5-year visit, with an annual change of $0.012 \pm 0.003 \quad(P=0.016)$. The MSQOL-54 score was $60.04 \pm 21.55$ at the baseline examination and $56.76 \pm 24.65$ at the 5 -year visit, with an annual change of $-0.65 \pm 0.21(P=0.003)$. Fifty-two (25.5\%) of the MS eyes had had at least 1 optic neuritis episode before beginning the study, and the mean time after these episodes to the beginning of the study was $7.74 \pm 6.28$ years. Age, gender, intraocular pressure, EDSS score, MSQOL-54 score, duration of disease, and time since last episode of optic neuritis are shown in Table 1.

Interferon $\beta$-1b was the most frequently prescribed drug for MS $(21.3 \%)$ at the beginning of the study, followed by subcutaneous interferon $\beta$-1a (15.7\%), intramuscular interferon $\beta$-1a $(11.4 \%)$, and glatiramer acetate (10.1\%). The percentage of patients not receiving any treatment at the beginning of the study was $38.0 \%$, and that at the end of the follow-up was $37.6 \%$. A total of 61 patients continued to receive their initially prescribed treatment throughout the study.

Significant thinning of all parameters of the peripapillary RNFL thickness and reduced macular volume based on OCT was detected at the 5-year follow-up visit in both patients and controls (except inferior RNFL thickness in healthy controls; Table 2).
Ganglion cell layer analysis of Cirrus OCT showed significant thinning of all but 2 parameters in MS patients (minimum inner plexiform layer plus GCL and inferotemporal quadrant). The change in fovea, temporal, and inferotemporal measurements of the GCL correlated significantly with the change in the EDSS score over the 5-year follow-up $(r=-0.341$ and $P=0.047$, $r=-0.497$ and $P=0.009$, and $r=-0.507$ and $P=0.002$, respectively).

Scanning laser polarimetry revealed a significant decrease in TSNIT average and a significant increase in the TSNIT standard deviation in the MS patient group, but these changes during the follow-up did not differ significantly compared with those of the healthy control group. A significant delay in the P100 wave latency in VEP was observed in the MS group during the follow-up and differed significantly from the control group throughout the study (Table 2).

In the MS group, annual mean RNFL thickness measured by OCT tended to decrease over the 5 years (Fig 1). The superior and temporal RNFL thicknesses exhibited greater changes between baseline and the 5-year follow-up in MS patients, with a decrease in annual mean thickness of $2.32 \mu \mathrm{m}(2.0 \%)$ and $2.18 \mu \mathrm{m}$ (3.4\%), respectively $(P<0.001$; Table 2 ; Fig 1$)$.

Changes in the structural variables revealed by OCT and SLP were not correlated significantly with changes in the functional ophthalmologic variables over the 5-year follow-up (best-corrected visual acuity, chromatic vision, visual field). The change in the temporal and average RNFL sectors based on OCT was moderately negatively correlated with the EDSS score over the 5-year follow-up $(r=-0.426$ and $P=0.007$, and $r=-0.398$ and $P=0.010$, respectively). In addition, the change in the temporal RNFL sector based on OCT was correlated moderately positively with the change in the MSQOL-54 score over the 5-year follow-up $(r=0.533$ and $P=0.031)$. The change in SLP parameters was not correlated significantly with the neurologic scale scores. The change in the inferior and average RNFL sectors based on OCT was correlated moderately with the change in the inferior and TSNIT averages of SLP over the 5-year follow-up $(r=0.701$ and $P=0.021$, and $r=0.742$ and $P=0.009$, respectively).

Changes in the functional and structural parameters over the 5-year follow-up were compared between eyes with and without a

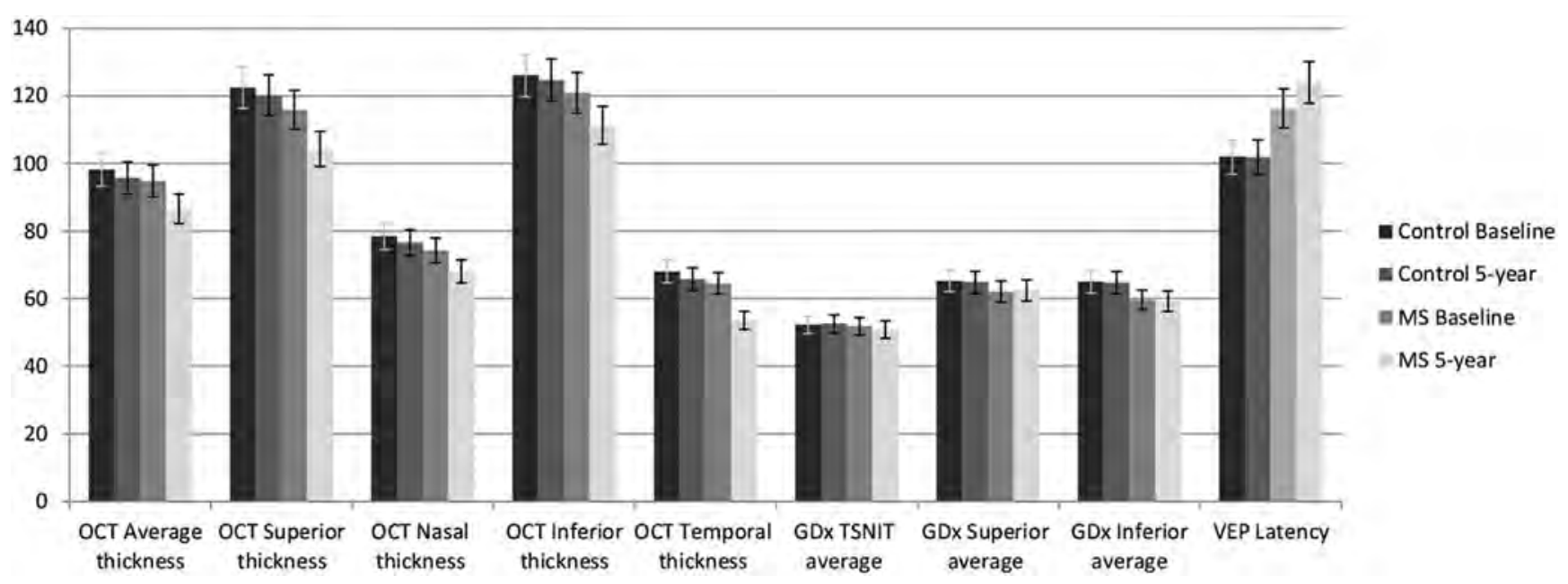

Figure 1. The mean retinal nerve fiber layer thickness measured by optical coherence tomography (OCT) and scanning laser polarimetry (SLP) and the mean P100 wave latency in visual evoked potentials (VEPs) in healthy controls and multiple sclerosis (MS) patients during 5 years of follow-up. The MS group showed more thinning of the retinal nerve fiber layer thickness during the follow-up and higher delays in the P100 wave latency. TSNIT $=$ temporal-superior-nasal-inferior-temporal. 
Table 3. Functional and Structural Parameters Obtained at Baseline and 5-Year Examinations in Multiple Sclerosis Patients with and without Optic Neuritis Antecedent and Comparisons of the Change in Each Group (Paired Student $t$ test), between Both Groups (Multiple Sclerosis Patients with and without Optic Neuritis; Student $t$ test), and between Both Groups and Healthy Controls (Analysis of Variance)

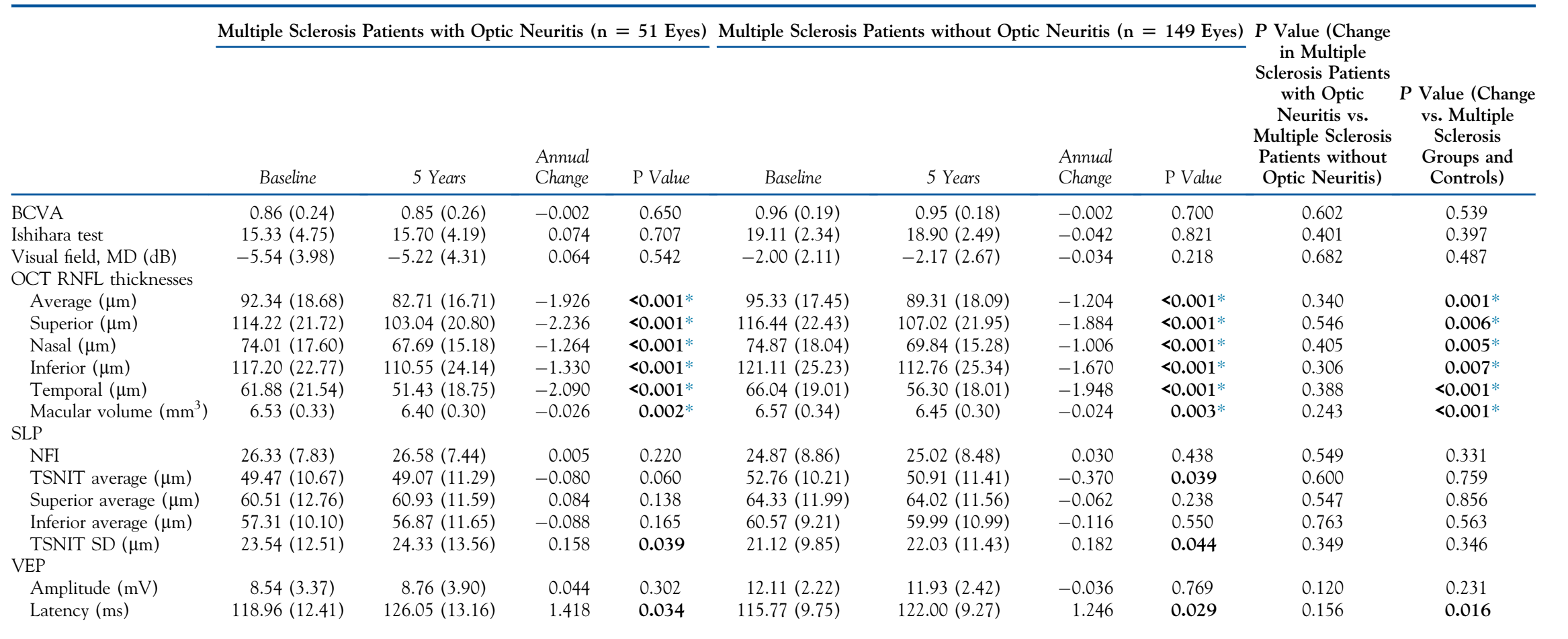

$\mathrm{BCVA}=$ best-corrected visual acuity; $\mathrm{MD}=$ mean deviation; $\mathrm{NFI}=$ nerve fiber indicator; $\mathrm{OCT}=$ optical coherence tomography; $\mathrm{SD}=$ standard deviation; $\mathrm{RNFL}=$ retinal nerve fiber layer; $\mathrm{SLP}=$ scanning laser polarimetry; TSNIT = temporal-superior-nasal-inferior-temporal; VEP = visual evoked potential.

Data are mean $(\mathrm{SD})$ unless otherwise indicated. $P<0.05$ was considered to indicate statistical significance.

*Statistical significance using Bonferroni's correction for multiple comparisons. All statistical differences in change between 3 groups using analysis of variance showed differences between multiple sclerosi eyes with or without optic neuritis antecedent and healthy controls, but not between multiple sclerosis eyes with optic neuritis antecedent and multiple sclerosis eyes without optic neuritis antecedent. 


\section{Ophthalmology Volume $\mathbf{-}$, Number $\mathbf{-}$, Month 2017}

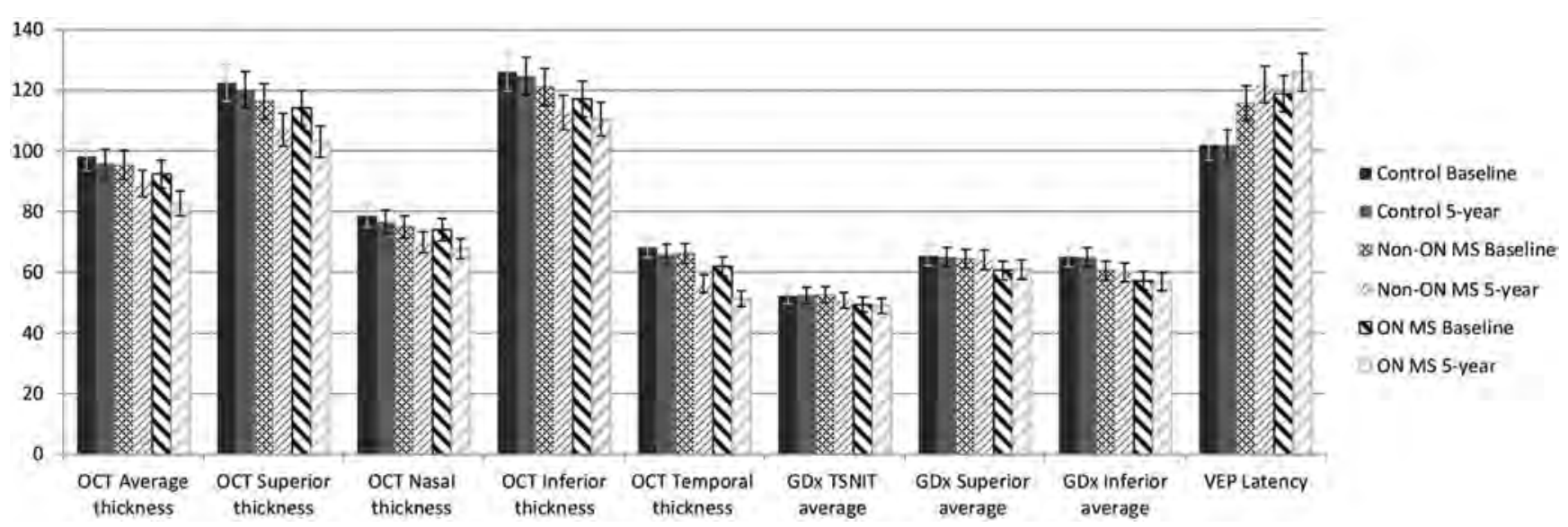

Figure 2. The mean retinal nerve fiber layer thickness measured by optical coherence tomography (OCT) and scanning laser polarimetry (SLP) and the mean P100 wave latency in visual evoked potentials (VEPs) in the 3 groups (healthy controls, multiple sclerosis [MS] patients without prior optic neuritis episode, and MS patients with prior optic neuritis episode) during 5 years of follow-up. Multiple sclerosis patients showed more shrinking of the retinal nerve fiber layer thicknesses during the follow-up and a greater delay in the P100 wave latency compared with healthy controls, but the changes were similar between both groups of MS patients (with and without prior optic neuritis episode). ON = optic neuritis; TSNIT = temporal-superior-nasal-inferior-temporal.

prior optic neuritis episode and with healthy controls. Both groups of patients exhibited a greater decrease in the RNFL parameters and greater increase in the VEP latency than healthy controls, but no differences were detected between eyes with and without prior optic neuritis (Table 3; Fig 2).

The mean annual change was compared with the change during the first year of the study in the MS cohort and no differences were detected. A greater decrease of structural variables revealed by OCT and SLP and a greater increase of VEP latency in the MS group during the 5-year follow-up were confirmed using the mean of eyes with nonoptic neuritis in the MS group (Table 4) to account for intereye within-patient dependencies. ${ }^{22}$ All the significant correlations described above were confirmed using the mean of eyes with nonoptic neuritis in the MS group.

\section{Discussion}

The findings of the present study based on OCT, SLP, and VEP indicate that quantification of RNFL thinning in patients with MS over a 5-year period can be used as a biomarker of disease progression and impaired QOL. Retinal nerve fiber layer thinning occurs not only in eyes with a prior episode of optic neuritis, but also in patients with MS with no history of acute optic neuritis. ${ }^{15,23}$ Retinal nerve fiber layer thickness measurements by OCT are considered a useful indirect marker of the progression of brain atrophy in patients with MS. ${ }^{2,16,24}$ Optical coherence tomography also is used to measure macular volume. The macula comprises ganglion cell bodies, and thus macular volume could be a useful indicator of axonal loss resulting from neuronal degeneration. ${ }^{25}$

In VEP, the P100 wave latency is highly diagnostic in patients with MS. An episode of optic neuritis can result in a delay in the P100 latency, with the delay lasting for years. ${ }^{11}$ In this study, the P100 latency increased over the 5-year follow-up because of MS progression and the loss of visual pathway function in the MS patients. The lack of a reduction in VEP amplitude despite thinning of the RNFL as assessed by OCT may be the result of small signals from the optic nerve (that may be amplified in the cortex, essentially obscuring or possibly compensating for small differences in input). Interestingly, the control group also showed a nonsignificant increase in the P100 latency, which may be the result of the variability of the device. Thus, although the VEP P100 wave latency is useful for monitoring MS progression, it is less sensitive than the OCT measurements. In our study, we found a very small decrease in the VEP amplitude and latency in the healthy controls, which was not significant, and we consider that the VEP variability explains the small change during the 5-year follow-up.

Functional parameters did not show a significant change during the follow-up, suggesting that the RNFL loss detected by OCT was not severe enough to affect functionality or that compensatory mechanisms could attenuate the neurodegenerating effects and avoid relevant clinical deficits measured by visual acuity, visual field, or color vision.

This is a prospective study with a 5-year follow up in MS and healthy controls using OCT, SLP, and VEP. Axonal loss in the RNFL was demonstrated in both healthy controls and MS patients, but was greater in the MS patients. Consistent with previous studies, MS patients with and without a prior optic neuritis episode in the present study exhibited similar RNFL thinning. ${ }^{15,23,26,27}$ These findings indicate that a prior optic neuritis episode is not a risk factor for higher axonal loss in the optic nerve. Our results confirmed the findings by Herrero et $\mathrm{al}^{26}$ of a progressive decrease in RNFL thicknesses and macular volume by OCT in MS patients compared with healthy controls. However, Herrero et al found an annual reduction of $0.152 \mu \mathrm{m}$ in the RNFL average thickness of the MS population, whereas we registered a greater decrease (an annual reduction, $1.642 \mu \mathrm{m}$ ). In the study by Herrero et al, patients were followed up only for 3 years. Balk et $\mathrm{al}^{27}$ found that the RNFL decrease is independent of previous episodes of optic neuritis, which is similar to our findings. 
Table 4. Functional and Structural Parameters Obtained at Baseline and 5-Year Examinations in Multiple Sclerosis Patients Using the Mean of Both Eyes in Nonoptic Neuritis Eyes and in Healthy Controls and Comparison of the Change in Each Group and between Both Groups (Multiple Sclerosis Patients Using the Mean of Both Eyes per Patient vs. Healthy Controls)

\begin{tabular}{|c|c|c|c|c|c|c|c|c|c|}
\hline & \multicolumn{4}{|c|}{ Multiple Sclerosis Patients Using Mean of Both Eyes in Nonoptic Neuritis Patients $(\mathrm{n}=125)$} & \multicolumn{4}{|c|}{ Healthy Controls $(\mathrm{n}=100)$} & \multirow{2}{*}{$\begin{array}{c}\text { P Value (Change } \\
\text { in Multiple Sclerosis } \\
\text { Patients vs. Healthy } \\
\text { Controls) }\end{array}$} \\
\hline & Baseline & 5 Years & $\begin{array}{l}\text { Annual } \\
\text { Change }\end{array}$ & P Value & Baseline & 5 Years & $\begin{array}{l}\text { Annual } \\
\text { Change }\end{array}$ & P Value & \\
\hline BCVA & $0.91(0.20)$ & $0.90(0.21)$ & -0.002 & 0.441 & $0.98(0.04)$ & $0.99(0.02)$ & 0.002 & 0.549 & 0.236 \\
\hline Ishihara test & $18.04(3.98)$ & $17.91(3.65)$ & -0.026 & 0.576 & $19.40(2.81)$ & $19.38(2.74)$ & -0.004 & 0.699 & 0.435 \\
\hline Visual field, MD (dB) & $-3.09(3.01)$ & $-3.12(3.87)$ & 0.006 & 0.670 & $0.31(1.41)$ & $0.29(1.31)$ & -0.004 & 0.105 & 0.611 \\
\hline \multicolumn{10}{|l|}{ OCT RNFL thicknesses } \\
\hline Average $(\mu \mathrm{m})$ & $94.43(15.91)$ & $86.75(16.23)$ & -1.536 & $<0.001 *$ & $98.21(10.50)$ & $95.79(9.53)$ & -0.484 & 0.032 & $0.003 *$ \\
\hline Superior $(\mu \mathrm{m})$ & $115.93(21.66)$ & $104.30(20.09)$ & -2.326 & $0.001 *$ & $122.49(16.93)$ & $120.27(14.93)$ & -0.444 & 0.035 & $0.007 *$ \\
\hline Nasal $(\mu \mathrm{m})$ & $74.32(20.29)$ & $68.07(18.41)$ & -1.250 & 0.002 & $78.50(21.76)$ & $76.59(10.70)$ & -0.382 & 0.044 & $0.004 *$ \\
\hline Inferior $(\mu \mathrm{m})$ & $119.59(21.54)$ & $111.65(21.55)$ & -1.588 & $<0.001 *$ & $126.10(18.36)$ & $124.73(17.94)$ & -0.274 & 0.075 & $0.009^{*}$ \\
\hline Temporal $(\mu \mathrm{m})$ & $64.49(20.19)$ & $53.64(17.76)$ & -2.170 & $<0.001 *$ & $68.14(9.82)$ & $65.87(9.56)$ & -0.454 & 0.028 & $0.002 *$ \\
\hline Macular volume $\left(\mathrm{mm}^{3}\right)$ & $6.54(0.34)$ & $6.43(0.32)$ & -0.022 & $<0.001 *$ & $8.29(0.44)$ & $8.23(0.55)$ & -0.012 & $0.009^{*}$ & $0.005^{*}$ \\
\hline \multicolumn{10}{|l|}{ OCT GCL analysis $(\mu \mathrm{m})$} \\
\hline Average IPL+GCL & $78.47(3.62)$ & $74.93(3.70)$ & -0.708 & $0.003 *$ & $83.12(3.20)$ & $81.56(3.43)$ & -0.312 & 0.079 & $0.007 *$ \\
\hline Minimum IPL+GCL & $79.12(4.87)$ & $77.54(3.54)$ & -0.316 & 0.301 & $81.23(3.69)$ & $80.00(3.11)$ & -0.246 & 0.106 & 0.110 \\
\hline Fovea & $253.01(9.43)$ & $249.03(9.00)$ & -0.796 & $0.008^{*}$ & $256.12(7.98)$ & $253.38(8.21)$ & -0.548 & 0.014 & 0.006 \\
\hline Inferior & $80.70(3.11)$ & $78.12(3.44)$ & -0.516 & 0.023 & $82.45(3.33)$ & $80.66(2.98)$ & -0.358 & 0.056 & 0.309 \\
\hline Inferonasal & $83.04(3.70)$ & $81.11(4.76)$ & -0.386 & 0.453 & $84.54(4.34)$ & $82.32(3.45)$ & -0.444 & 0.035 & 0.488 \\
\hline Nasal & $84.69(3.35)$ & $80.87(4.22)$ & -0.764 & 0.011 & $85.25(4.21)$ & $83.22(3.87)$ & -0.406 & 0.078 & 0.680 \\
\hline Superior & $80.93(3.24)$ & $77.50(4.57)$ & -0.686 & 0.031 & $83.21(3.66)$ & $80.98(3.27)$ & -0.446 & 0.028 & 0.047 \\
\hline Inferotemporal & $78.77(3.64)$ & $74.30(4.01)$ & -0.894 & $0.006^{*}$ & $82.02(3.41)$ & $80.56(3.70)$ & -0.292 & 0.230 & 0.008 \\
\hline Temporal & $78.88(3.37)$ & $74.12(4.33)$ & -0.952 & $0.003 *$ & $81.11(3.93)$ & $79.82(4.11)$ & -0.258 & 0.177 & 0.007 \\
\hline \multicolumn{10}{|c|}{ (1.02 (1.11) } \\
\hline NFI & $25.19(9.18)$ & $25.46(8.65)$ & 0.054 & 0.541 & $18.70(6.87)$ & $19.04(7.80)$ & 0.068 & 0.349 & 0.854 \\
\hline TSNIT average $(\mu \mathrm{m})$ & $51.85(9.13)$ & $50.92(12.40)$ & -0.014 & 0.904 & $52.33(6.01)$ & $52.61(7.71)$ & 0.056 & 0.451 & 0.550 \\
\hline Superior average $(\mu \mathrm{m})$ & $62.16(10.76)$ & $62.47(11.61)$ & 0.062 & 0.332 & $65.32(5.98)$ & $64.87(5.50)$ & -0.090 & 0.559 & 0.256 \\
\hline Inferior average $(\mu \mathrm{m})$ & $59.66(9.67)$ & $59.33(10.12)$ & -0.066 & 0.421 & $65.00(7.09)$ & $64.76(6.96)$ & -0.048 & 0.683 & 0.587 \\
\hline TSNIT SD $(\mu \mathrm{m})$ & $21.96(4.88)$ & $23.13(5.31)$ & 0.234 & 0.040 & $20.03(4.21)$ & $20.61(4.16)$ & 0.116 & 0.078 & 0.101 \\
\hline \multicolumn{10}{|l|}{ VEP } \\
\hline Amplitude (mV) & $11.19(2.80)$ & $10.90(3.44)$ & -0.058 & 0.412 & $14.54(2.67)$ & $14.49(2.55)$ & -0.010 & 0.449 & 0.254 \\
\hline Latency (ms) & $117.11(12.93)$ & $123.43(12.99)$ & 1.264 & $0.006^{*}$ & $102.03(7.49)$ & $101.89(6.90)$ & -0.028 & 0.618 & 0.030 \\
\hline
\end{tabular}

$\mathrm{BCVA}=$ best-corrected visual acuity; $\mathrm{GCL}=$ ganglion cell layer; $\mathrm{IPL}=$ inner plexiform layer; $\mathrm{MD}=$ mean deviation; $\mathrm{NFI}=$ nerve fiber indicator; $\mathrm{OCT}=$ optical coherence tomography; $\mathrm{RNFL}=$ retinal nerve fiber layer; $\mathrm{SD}=$ standard deviation; SLP = scanning laser polarimetry; TSNIT = temporal-superior-nasal-inferior - temporal; VEP = visual evoked potential.

Data are mean $(\mathrm{SD})$ unless otherwise indicated. $P<0.05$ was considered to indicate statistical significance.

*Statistical significance using Bonferroni's correction for multiple comparisons. 
Although we detected no significant correlation between the changes in SLP parameters and MS progression, the potential ability of SLP to identify changes in axonal cytoskeletal processes before neuronal loss and peripapillary RNFL axon bundles has been described by other authors. ${ }^{28}$ We found an association between changes in SLP and OCT in our study, and thus it may be that increasing the sample size would demonstrate that SLP changes correlate with disease progression.

Recent studies demonstrate the usefulness of monitoring peripapillary RNFL thickness by OCT for predicting the likelihood of increased disability in MS patients over time ${ }^{17}$ and for predicting a decrease in the quality of life. ${ }^{21}$ Our findings are in accordance with these previous reports, because we found an association between RNFL thinning and an increased EDSS score (RNFL reduction in the temporal sector or average thicknesses was associated with higher disability in these patients) and between a decrease in the temporal RNFL sector and a decrease in the MSQOL-54 score (patients with higher temporal RNFL sector thinning show impaired QOL).

In addition, our study demonstrates an association between decreases in the fovea, temporal, and inferotemporal measurements of the GCL provided by OCT and an increase in the EDSS score over 5 years. As recent studies suggest, changes in GCL are even more appropriate markers of clinical neurodegeneration in the visual pathway. ${ }^{29,30}$

Each eye was analyzed separately in this study because the eyes can be affected differentially, especially in patients with unilateral episodes of optic neuritis. Moreover, OCT devices compare the 3-dimensional profile of the RNFL thickness of each eye to a normative database. Finally, in MS patients, a reduction in the macular volume correlates with neuronal necrosis, demyelination, and axonal damage. ${ }^{21}$ The loss of ganglion cells also leads to a reduction of the macular volume (independently in each eye). ${ }^{2,6,31-33}$ However, some authors consider the inclusion of only 1 eye of each patient adequate for statistical analysis because RNFL measurements correlate significantly between the 2 eyes. In our study, we reanalyzed the data using the mean of eyes with nonoptic neuritis in the MS group (Table 4) to account for intereye within-patient dependencies, as recommended by the International Multiple Sclerosis Visual consortium, ${ }^{22}$ and the results of this analysis confirmed the progressive axonal loss in MS patients compared with healthy controls, and the correlation between the increase in the EDSS and temporal and superior RNFL thinning detected by OCT, and between decreased QOL and temporal RNFL thinning measured by OCT.

Previous studies indicate that a time lapse of 6 months after an optic neuritis episode is required to detect retrograde degeneration based on measurements made using digital image analysis techniques. ${ }^{31}$ Here we excluded patients with an optic neuritis episode during the 6 months preceding the study, so all participants were considered to have stable MS. We might have detected greater differences if we had included patients with active acute relapsing MS episodes in the analysis. However, we excluded these patients because the purpose of the study was to assess only axonal damage secondary to the progression of MS. The RNFL changes we observed were the result of only MS-related chronic neurodegeneration, and not of the acute heavy axonal loss found in acute relapsing MS episodes. The change in the evaluated parameters during the first few years was similar to the mean annual change, suggesting that progressive RNFL loss is continuous.

A potential limitation of this study is that our population mainly comprised a mildly affected relapsing-remitting MS cohort with low EDSS scores, high rates of no or first-line therapy, stable clinical disease activity without relapse within 5 years of observation, and a high mean age and disease duration at inclusion, because our intention was to reveal neurodegeneration independently of disease activity, so RNFL thinning may be higher in an MS cohort with normal disease activity. This should be kept in mind when interpreting the clinical findings. In conclusion, OCT measurements of RNFL thickness are useful for detecting axonal damage in MS patients and can be used to monitor disease progression and changes in QOL.

\section{References}

1. Fisher JB, Jacobs DA, Markowitz CE, et al. Relation of visual function to retinal nerve fiber layer thickness in multiple sclerosis. Ophthalmology. 2006;113:324-332.

2. Gordon-Lipkin E, Chodkowski B, Reich DS, et al. Retinal nerve fiber layer is associated with brain atrophy in multiple sclerosis. Neurology. 2007;69:1603-1609.

3. Garcia-Martin E, Pueyo V, Pinilla I, et al. Fourier-domain OCT in multiple sclerosis patients: reproducibility and ability to detect retinal nerve fiber layer atrophy. Invest Ophthalmol Vis Sci. 2011;52:4124-4131.

4. Khanifar AA, Parlitsis GJ, Ehrlich JR, et al. Retinal nerve fiber layer evaluation in multiple sclerosis with spectral domain optical coherence tomography. Clin Ophthalmol. 2010;4: 1007-1013.

5. Sergott RC. Optical coherence tomography: measuring in-vivo axonal survival and neuroprotection in multiple sclerosis and optic neuritis. Curr Opin Ophthalmol. 2005;16: 346-350.

6. Zaveri MS, Conger A, Salter A, et al. Retinal imaging by laser polarimetry and optical coherence tomography evidence of axonal degeneration in multiple sclerosis. Arch Neurol. 2008;65:924-928.

7. Gundogan FC, Demirkaya S, Sobaci G. Is optical coherence tomography really a new biomarker candidate in multiple sclerosis? A structural and functional evaluation. Invest Ophthalmol Vis Sci. 2007;48:5773-5781.

8. Garcia-Martin E, Pueyo V, Martin J, et al. Progressive changes in the retinal nerve fiber layer in patients with multiple sclerosis. Eur J Ophthalmol. 2010;20:167-173.

9. Trip SA, Schlottmann PG, Jones SJ, et al. Retinal nerve fiber layer axonal loss and visual dysfunction in optic neuritis. Ann Neurol. 2005;58:383-391.

10. Weinstock-Guttman B, Baier M, Stockton R, et al. Pattern reversal visual evoked potentials as a measure of visual pathway pathology in multiple sclerosis. Mult Scler. 2003;9: 529-534. 


\section{Garcia-Martin et al • Progressive Degeneration in MS}

11. Rodriguez-Mena D, Almarcegui C, Dolz I, et al. Electrophysiologic evaluation of the visual pathway in patients with multiple sclerosis. J Clin Neurophysiol. 2013;30:376-381.

12. Henderson AP, Trip SA, Schlottmann PG, et al. A preliminary longitudinal study of the retinal nerve fiber layer in progressive multiple sclerosis. J Neurol. 2010;257:1083-1091.

13. Ratchford JN, Quigg ME, Conger A, et al. Optical coherence tomography helps differentiate neuromyelitis optica and MS optic neuropathies. Neurology. 2009;73:302-308.

14. Syc SB, Warner CV, Hiremath GS, et al. Reproducibility of high-resolution optical coherence tomography in multiple sclerosis. Mult Scler. 2010;16:829-839.

15. Garcia-Martin E, Pueyo V, Ara JR, et al. Effect of optic neuritis on progressive axonal damage in multiple sclerosis patients. Mult Scler. 2011;17:830-837.

16. Frohman EM, Dwyer MG, Frohman T, et al. Relationship of optic nerve and brain conventional and non-conventional MRI measures and retinal nerve fiber layer thickness, as assessed by OCT and GDx: a pilot study. J Neurol Sci. 2009;282:96-105.

17. Martinez-Lapiscina EH, Arnow S, Wilson JA, et al. Retinal thickness measured with optical coherence tomography and risk of disability worsening in multiple sclerosis: a cohort study. Lancet Neurol. 2016;15:574-584.

18. Polman CH, Reingold SC, Banwell B, et al. Diagnostic criteria for multiple sclerosis: 2010 revisions to the McDonald criteria. Ann Neurol. 2011;69:292-302.

19. Vickrey BG, Hays RD, Harooni R, et al. A health-related quality of life for multiple sclerosis. Qual Life Res. 1995;4: 187-206.

20. Meyers AR, Gage H, Hendricks A. Health-related quality of life in neurology. Arch Neurol. 2000;57:1224-1227.

21. Garcia-Martin E, Rodriguez-Mena D, Herrero R, et al. Neuroophthalmologic evaluation, quality of life, and functional disability in patients with MS. Neurology. 2013;81:76-83.

22. Cruz-Herranz A, Balk LJ, Oberwahrenbrock T, et al. The APOSTEL recommendations for reporting quantitative optical coherence tomography studies. Neurology. 2016;86: 2303-2309.

\section{Footnotes and Financial Disclosures}

Originally received: September 21, 2016.

Final revision: January 3, 2017.

Accepted: January 3, 2017.

Available online: $\mathbf{\square} \mathbf{\square}$.

Manuscript no. 2016-387.

${ }^{1}$ Department of Ophthalmology, Miguel Servet University Hospital, Zaragoza, Spain.

${ }^{2}$ Aragon Institute for Health Research (Instituto de Investigación Sanitario [IIS] Aragon), University of Zaragoza, Zaragoza, Spain.

${ }^{3}$ Department of Neurology, Miguel Servet University Hospital, Zaragoza, Spain.

${ }^{4}$ Department of Neurophysiology, Miguel Servet University Hospital, Zaragoza, Spain.

Financial Disclosure(s):

The author(s) have no proprietary or commercial interest in any materials discussed in this article.

Author Contributions:

Conception and design: Garcia-Martin, Polo, Larrosa
23. Talman LS, Bisker ER, Sackel DJ, et al. Longitudinal study of vision and retinal nerve fiber layer thickness in multiple sclerosis. Ann Neurol. 2010;67:749-760.

24. Dörr J, Wernecke KD, Bock M, et al. Association of retinal and macular damage with brain atrophy in multiple sclerosis. PLoS One. 2011;6:e18132.

25. Burkholder BM, Osborne B, Loguidice MJ, et al. Macular volume determined by optical coherence tomography as a measure of neuronal loss in multiple sclerosis. Arch Neurol. 2009;66:1366-1372.

26. Herrero R, Garcia-Martin E, Almarcegui C, et al. Progressive degeneration of the retinal nerve fiber layer in patients with multiple sclerosis. Invest Ophthalmol Vis Sci. 2012;53(13): 8344-8349.

27. Balk LJ, Cruz-Herranz A, Albrech P, et al. Timing of retinal neuronal and axonal loss in MS: a longitudinal OCT study. J Neurol. 2016;263(7):1323-1331.

28. Fortune B, Burgoyne CF, Cull GA, et al. Structural and functional abnormalities of retinal ganglion cells measured in vivo at the onset of optic nerve head surface change in experimental glaucoma. Invest Ophthalmol Vis Sci. 2012;53(7):3939-3950.

29. Saidha S, Al-Louzi O, Ratchford JN, et al. Optical coherence tomography reflects brain atrophy in multiple sclerosis: a fouryear study. Ann Neurol. 2015;78(5):801-813.

30. Saidha S, Sotirchos ES, Ibrahim MA, et al. Microcystic macular oedema, thickness of the inner nuclear layer of the retina, and disease characteristics in multiple sclerosis: a retrospective study. Lancet Neurol. 2012;11(11):963-972.

31. Costello F, Coupland S, Hodge W, et al. Quantifying axonal loss after optic neuritis with optical coherence tomography. Ann Neurol. 2006;59:963-969.

32. Klistorner A, Arvind H, Garrick R, et al. Interrelationship of optical coherence tomography and multifocal visual-evoked potentials after optic neuritis. Invest Ophthalmol Vis Sci. 2010;51:2770-2777.

33. Grazioli E, Zivadinov R, Weinstock-Guttman B, et al. Retinal nerve fiber layer thickness is associated with brain MRI outcomes in multiple sclerosis. J Neurol Sci. 2008;268:12-17.
Analysis and interpretation: Garcia-Martin, Polo, Larrosa, Pablo, Satue

Data collection: Garcia-Martin, Ara, Martin, Almarcegui, Dolz, Vilades, Gil-Arribas, Fernandez, Satue

Obtained funding: none

Overall responsibility: Garcia-Martin, Ara, Martin, Almarcegui, Dolz, Satue

Abbreviations and Acronyms:

$\mathbf{C z}=$ Z-center, equidistant point between both ear points; EDSS $=$ Expanded Disability Status Scale; GCL $=$ ganglion cell layer MS = multiple sclerosis; MSQOL-54 $=54$-item Multiple Sclerosis Quality of Life Scale; OCT = optical coherence tomography; $\mathbf{Q O L}=$ quality of life; $\mathbf{R N F L}=$ retinal nerve fiber layer; $\mathbf{S L P}=$ scanning laser polarimetry; TSNIT $=$ superior-nasal-inferior-temporal; VEP = visual evoked potential

Correspondence:

Elena Garcia-Martin, PhD, C/ Padre Arrupe, Consultas Externas de Oftalmología Edificio de consultas externas, Planta 1, 50009 Zaragoza, Spain. E-mail: egmvivax@yahoo.com. 